\title{
Specific Heat Anomalies in Solids Described by a Multilevel Model
}

\author{
Mariano de Souza $^{1,3}$ (iD $\cdot$ Ricardo Paupitz $^{1} \cdot$ Antonio Seridonio $^{1,2} \cdot$ Roberto E. $_{\text {Lagos }}{ }^{1}$
}

Received: 23 July 2015 / Published online: 19 February 2016

(c) Sociedade Brasileira de Física 2016

\begin{abstract}
In the field of condensed matter physics, specific heat measurements can be considered as a pivotal experimental technique for characterizing the fundamental excitations involved in a certain phase transition. Indeed, phase transitions involving spin (de Souza et al. Phys. B Condens. Matter 404, 494 (2009) and Manna et al. Phys. Rev. Lett. 104, 016403 (2010)), charge (Pregelj et al. Phys. Rev. B 82, 144438 (2010)), lattice (Jesche et al. Phys. Rev. B 81, 134525 (2010)) (phonons) and orbital degrees of freedom, the interplay between ferromagnetism and superconductivity (Jesche et al. Phys. Rev. B 86, 020501 (2012)), Schottkylike anomalies in doped compounds (Lagos et al. Phys. C Supercond. 309, 170 (1998)), electronic levels in finite correlated systems (Macedo and Lagos J. Magn. Magn. Mater. 226, 105 (2001)), among other features, can be captured by means of high-resolution calorimetry. Furthermore, the entropy change associated with a first-order phase transition, no matter its nature, can be directly obtained upon integrating the specific heat over $T$, i.e., $C(T) / T$, in the temperature range of interest. Here, we report on a detailed analysis of the two-peak specific heat anomalies observed in several materials. Employing a simple multilevel model,
\end{abstract}

Mariano de Souza

mariano@rc.unesp.br

1 Departamento de Física, IGCE, Unesp - Univ Estadual Paulista, 13506-900, Rio Claro, SP, Brazil

2 Departamento de Física e Química, Unesp - Univ Estadual Paulista, 15385-000, Ilha Solteira, SP, Brazil

3 Present address: Institute of Semiconductor and Solid State Physics, Johannes Kepler University Linz, Linz, Austria varying the spacing between the energy levels $\Delta_{i}=\left(E_{i}-\right.$ $\left.E_{0}\right)$ and the degeneracy of each energy level $g_{i}$, we derive the required conditions for the appearance of such anomalies. Our findings indicate that a ratio of $\Delta_{2} / \Delta_{1} \approx 10$ between the energy levels and a high degeneracy of one of the energy levels define the two-peaks regime in the specific heat. Our approach accurately matches recent experimental results. Furthermore, using a mean-field approach, we calculate the specific heat of a degenerate Schottky-like system undergoing a ferromagnetic (FM) phase transition. Our results reveal that as the degeneracy is increased the Schottky maximum in the specific heat becomes narrow while the peak associated with the FM transition remains unaffected.

Keywords Specific heat · Partition function · Correlation effects

\section{Introduction}

In his seminal paper of 1907 [8], in order to explain the deviation of the specific heat of certain materials, like silicon, boron, and carbon, from the Dulong-Petit's law, Einstein proposed a model based on the assumption that all atoms in a solid vibrate independently from each other with the same eigen-frequency. It is worth mentioning that recently, one of us made use of Einstein's model to determine the eigenenergy of the counter-anions libration modes in a molecular conductor [9]. However, thought the success of the model proposed by Einstein, the theoretical description introduced by P. Debye in 1912 [10], constitutes the hallmark in the description of the phononic contribution to the specific heat in solids, see, e.g., [11]. Essentially, Debye's model is based 
on the hypothesis of a continuous isotropic solid. The dispersion relation is linear, i.e., the sound velocity is constant and isotropic, being a set of eigen-frequencies allowed to the oscillators. In the frame of Debye's model, the phononic specific heat at constant volume, $C_{p h, v}^{D}(T)$, reads:

$C_{p h, v}^{D}(T)=3\left(\frac{T}{\Theta_{D}}\right)^{3} \int_{0}^{x} \frac{x^{4} e^{x}}{\left(e^{x}-1\right)^{2}} d x$,

where $x=\Theta_{D} / T$ and $\Theta_{D}$ is the so-called Debye temperature. The behavior of the specific heat as a function of $T$, according to (1), well-known from textbooks, is shown in the main panel of Fig. 1. A particular behavior, usually not explicitly shown and discussed in textbooks [12], is observed by plotting $C / T$ versus $T$, cf. inset of Fig. 1 . The maximum centered at $0.28 \cdot \Theta_{D}$ corresponds to the inflexion point of (1) and manifests itself as a direct effect of the accessible energy levels upon increasing the temperature. In other words, as the temperature of a certain Debye's solid is increased the number of accessible energy levels is reduced and, as a consequence, the entropy variation rate is reduced. The latter indicates that for any system which obeys a Debye-like behavior, $\Theta_{D}$ can be directly estimated by plotting $C / T$ versus $T$. Similarly in Einstein's model, $C / T$ versus $T$ has a maximum centered at $0.38 \cdot \Theta_{E}$. A combined system of Debye and Einstein phonons can be studied provided the Einstein, and Debye temperature scales are not so close [13].

At low temperatures, namely for ordinary metals $T \simeq$ $\Theta_{D} / 50 \simeq$ liquid ${ }^{4} \mathrm{He}$ temperature, the following relation is valid [11]:

$C_{v}(T)=\gamma T+A T^{3}$,

where $\gamma$ is the Sommerfeld coefficient and $A=12 \pi^{4} / 5 \Theta_{D}^{3}$. Here, the Debye temperature can be directly obtained via $A$ parameter, whereas the effective mass $m^{*}$ of the charge carriers in a metal can be estimated by means of

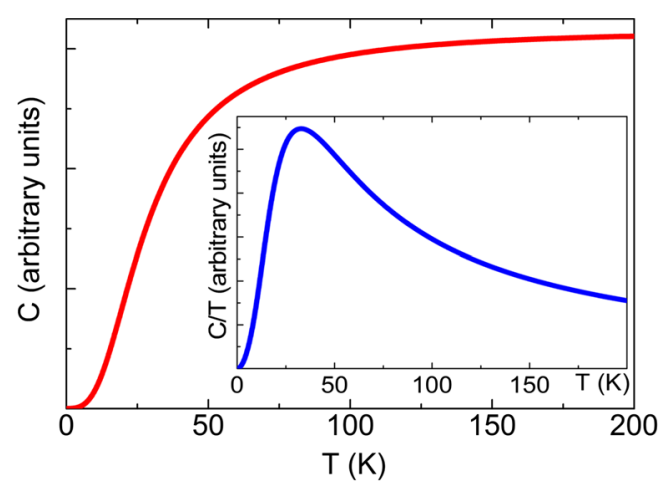

Fig. 1 Main panel: Specific heat $(C)$ as a function of temperature for an hypothetical Debye's solid (cf. (1)) with $\Theta_{D}=120 \mathrm{~K}$. Inset: $C / T$ versus $T$ showing a maximum centered at $T \simeq 0.28 \cdot \Theta_{D}$ the Sommerfeld coefficient [11]. The latter can be considered the smoking gun, for instance, when discovering materials with heavy-fermion-like behavior. Moreover, still considering the relevance of high-resolution calorimetry, combining $C_{p}(T)$ and the linear thermal expansion coefficient [14], $\alpha_{i}(T)$, and making use of the Ehrenfest theorem, see e.g. [11], the uniaxial-pressure dependence of the critical temperature for pressure applied along the $i$-axis for a second-order phase transition can be directly obtained. It is worth mentioning that in specific heat measurements the phononic contribution is usually disentangled from critical fluctuations by considering lattice contribution to the specific heat according with Debye's model [11]. In this regard, please refer to refs. $[4,15,16]$

For a general two-level system, separated by an energy gap $\Delta_{1}$, the specific heat is described by the following expression:

$C=\frac{\left(\beta \Delta_{1}\right)^{2} e^{-\beta \Delta_{1}}}{\left(1+e^{-\beta \Delta_{1}}\right)^{2}}$,

In such a system, the so-called Schottky anomaly manifests itself as a shallow maximum in the specific heat data as a function of temperature.

After this brief introduction, recalling some fundamental aspects related to specific heat measurements in solids, well-known from text books, see e.g. [11], below we present a multilevel model to describe systems which present multiple peaks in the specific heat data. Interestingly enough, such a simple model is capable to describe experimental electronic specific heat results of correlated electrons systems.

\section{Mathematical Modeling}

In the frame of the canonical ensemble, the sum over states, namely the partition function $(Z)$ of a system, is given by:

$Z \equiv \operatorname{Tr}\left(e^{-\beta \hat{H}}\right)=\sum_{n} g_{n} e^{-\beta E_{n}}$,

where $E_{n}$ refers to the $n^{\text {th }}$ energy level, i.e., the $n^{\text {th }}$ eigenvalue of the system's Hamiltonian $\hat{H}$ [17]. As a matter of fact, the partition function encodes all information of the physical system, so that once we know the energy eigenvalues of the physical system all thermodynamic observables can be calculated. This in turn is not the case if one has the system Hamiltonian in hand [18], being necessary to make its diagonalization to obtain $Z$ and then the physical quantities of interest. For the sake of simplicity, in order to describe the two-peak like anomalies observed experimentally in several materials (see below), we propose a multilevel model approach. This Ansatz enables us to obtain directly the thermodynamic quantities of interest. In other 
words, within such an approach, we work in the diagonal representation of the Hamiltonian $\hat{H}$ (cf. (4)). We start by considering a three-level model [19], with $\Delta_{1}=\left(E_{1}-E_{0}\right)$ and $\Delta_{2}=\left(E_{2}-E_{0}\right)$, being $E_{0}, E_{1}$, and $E_{2}$ defined as shown in Fig. 2, the partition sum reads

$$
Z=g_{0} e^{-\beta E_{0}}+g_{1} e^{-\beta\left(E_{0}+\Delta_{1}\right)}+g_{2} e^{-\beta\left(E_{0}+\Delta_{2}\right)},
$$

where $g_{i}$ indicates the degeneracy of each energy level $(i=$ $0,1,2)$. The assumption that the energy gaps $\Delta_{i}$, i.e., energy scales for a generic system, do not depend on the temperature is quite realistic. In this regard, we refer, for instance, to the Schottky model [11], where the energy gap $\Delta_{1}$ separating the two energy-levels is fixed and thus temperature independent.

Making use of (5), one can calculate the specific heat, employing the following well-known relations

$E=-\frac{\partial}{\partial \beta}(\ln Z)$

and

$C=-\beta^{2}\left(\frac{\partial E}{\partial \beta}\right)$

obtaining

$C=\beta^{2} \frac{\sum_{i} \sum_{j} g_{i} g_{j} \Delta_{i}\left(\Delta_{i}-\Delta_{j}\right) e^{-\beta\left(\Delta_{i}+\Delta_{j}\right)}}{\left[\sum_{i} g_{i} e^{-\beta \Delta_{i}}\right]^{2}}$,

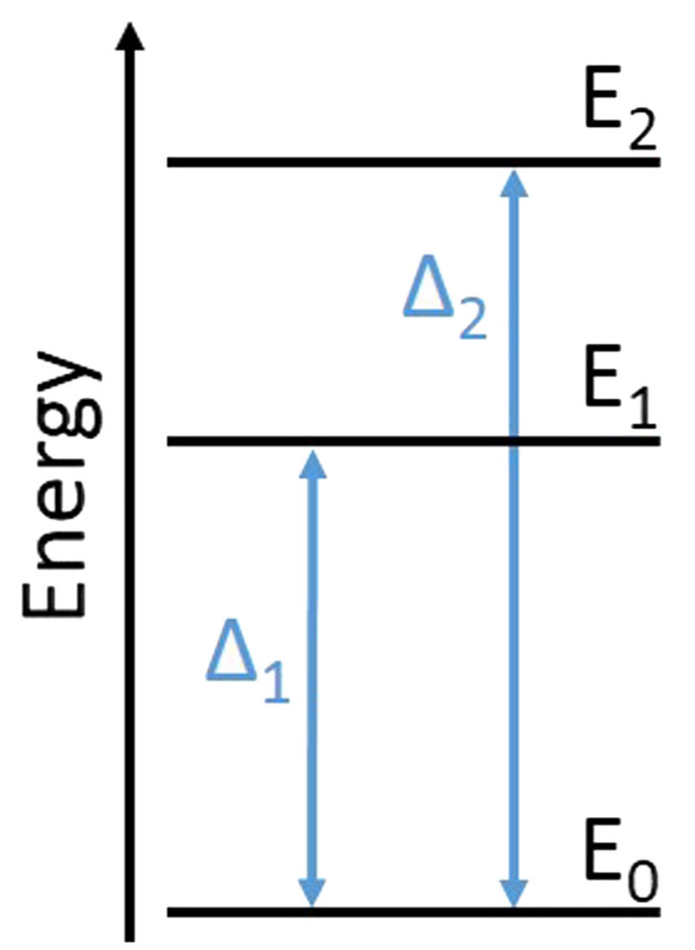

Fig. 2 Schematic representation of the energy levels $E_{0}, E_{1}$, and $E_{2}$ considered in the model (5). The excitation gaps $\Delta_{1}$ and $\Delta_{2}$ are defined as the energy difference relative to the ground state energy $\left(E_{0}\right)$. See discussion in the main text for details where $i, j=0,1,2$ and $\Delta_{0}=0$. Note that considering $j$ $=1$ in (8), the specific heat for the Schottky model (3) is nicely restored.

For the sake of completeness, we calculated the summations in (8) and present below the expression employed in our analysis:

$$
\begin{aligned}
C= & \beta^{2} \frac{g_{1} g_{0} \Delta_{1}^{2} e^{-\beta \Delta_{1}}+g_{0} g_{2} \Delta_{2}^{2} e^{-\beta \Delta_{2}}}{\left[g_{0}+g_{1} e^{-\beta \Delta_{1}}+g_{2} e^{-\beta \Delta_{2}}\right]^{2}} \\
& +\frac{g_{1} g_{2} e^{-\beta\left(\Delta_{1}+\Delta_{2}\right)}\left[\Delta_{1}\left(\Delta_{1}-\Delta_{2}\right)+\Delta_{2}\left(\Delta_{2}-\Delta_{1}\right)\right]}{\left[g_{0}+g_{1} e^{-\beta \Delta_{1}}+g_{2} e^{-\beta \Delta_{2}}\right]^{2}} .
\end{aligned}
$$

In the present model, the crucial feature refers to the relation between the energy scales of the two excitation gaps. The latter together with the degeneracies $g_{i}$ of the energy levels, define whether the system presents a Schottky-like behavior with a single peak/maximum in the specific heat or if it will exhibit two or even three (in this case a four energy levels is required) peaks or maxima. More specifically, one can say that in such cases two and three energy scales govern the physics of the system of interest.

\section{Results and Discussion}

In what follows, employing (9), we discuss the required conditions for the emergence of the two-peak anomalies in the specific heat. In Fig. 3, we present 3-dimensional (3D) plots of the specific heat as a function of temperature and degeneracy of the energy levels 1 (Fig. 3a) and 2 (Fig. 3b) and, specific heat as a function of temperature and the relative value of the energy levels 1 (Fig. 3c) and 2 (Fig. 3d) as well. The optimized parameters used in such set of fits, appropriate to give rise to the emergence of a double peak in the specific heat are $\Delta_{0}=0, \Delta_{1}=205 \mathrm{~J} / \mathrm{mol}, \Delta_{2}=$ $2327 \mathrm{~J} / \mathrm{mol}, g_{0}=1, g_{1}=2, g_{2}=4$, i.e., in each $3 \mathrm{D}$ plot one of these parameters was varied while the remaining ones were kept constant. For instance, in Fig. 3a, $T$ and $g_{1}$ were used as the variable parameters. Note that upon increasing the degeneracy $g_{1}$ the double peak in the specific heat gradually vanishes. A distinct situation is depicted in Fig. 3b, where the double peak in the specific heat appears as the degeneracy $g_{2}$ is increased. In Fig. $3 c$, d, we vary the temperature and the energy levels $\Delta_{1}$ and $\Delta_{2}$, respectively. The present findings indicate two distinct ways of catching a double-peak in the specific heat, i.e., either by varying the degeneracy or by increasing the energy levels $\Delta_{1}$ or $\Delta_{2}$. More specifically, Fig. $3 \mathrm{a} b$ indicates that at least one of the energy levels should be highly degenerated to give way to the double-peak in the specific heat. Similarly to the Schottky-like anomaly, this behavior suggest that the high degeneracy contribute dramatically to the specific heat over 

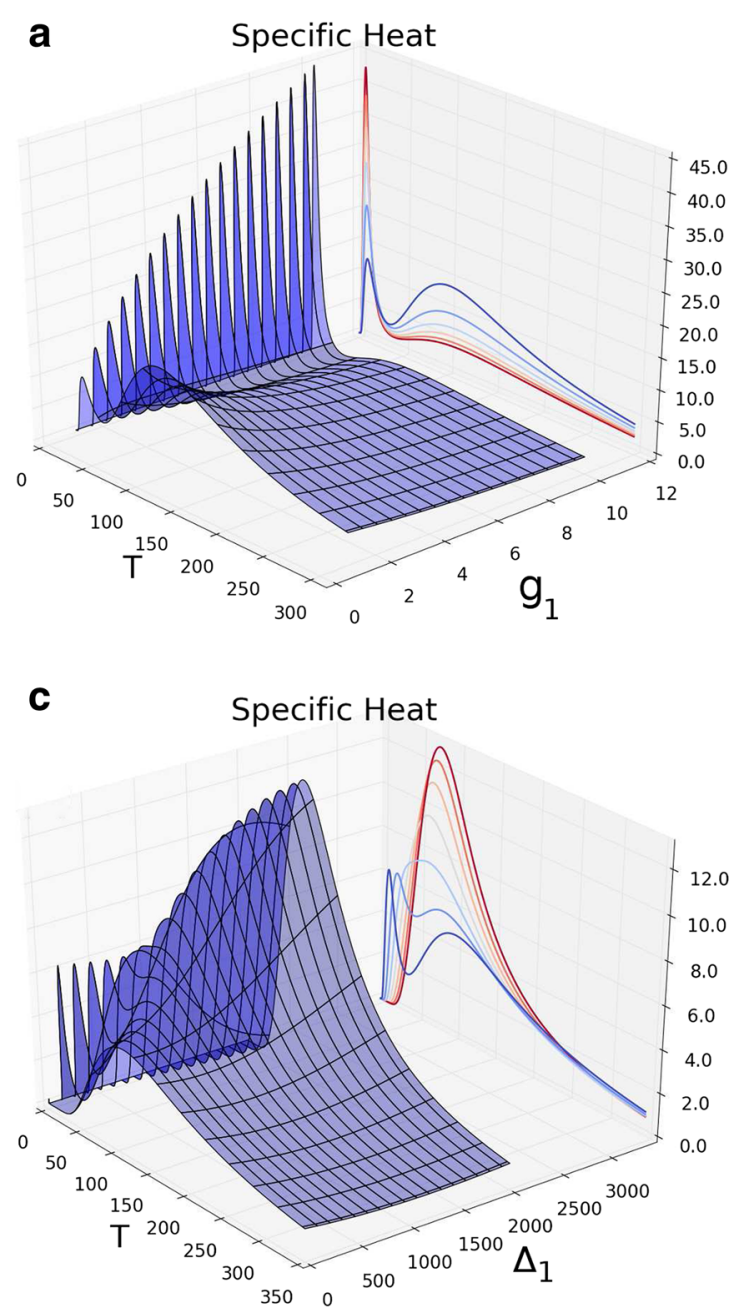

Fig. 3 Specific heat $(C)$ as a function of: a Temperature $(T)$ and degeneracy of the energy level 1 , labeled $g_{1} ; \mathbf{b} T$ and degeneracy of the energy level $2, g_{2} ; \mathbf{c} T$ and energy gap $\Delta_{1} ; \mathbf{d} T$ and energy gap $\Delta_{2}$.

a quite restricted $T$-window. Such a behavior can be easily understood in terms of the system entropy: once the number of accessible states is increased and this is the physical situation of a highly degenerated generic system or energy level, the entropy of the system is increased and, as a consequence, a double-peak structure shows up in the specific heat, since the entropy of the system is proportional to the area and can be estimated via $\Delta S=\int_{T_{a}}^{T_{b}} C / T d T$, where $T_{a}$ and $T_{b}$ indicate two distinct temperatures in the range of interest. In other words, the system entropy is increased at the expense of the satellite peak in the specific heat. Nevertheless, as discussed above, the specific heat is a bulk property. Thus, in order to map, the energy levels of a generic system and its degeneracy, microscopic experimental techniques like electron spin and magnetic resonance [20], for instance, are required. We stress that the two-maxima observed in the specific heat, obtained from the above-discussed Ansatz, is a universal signature of any system with two distinct
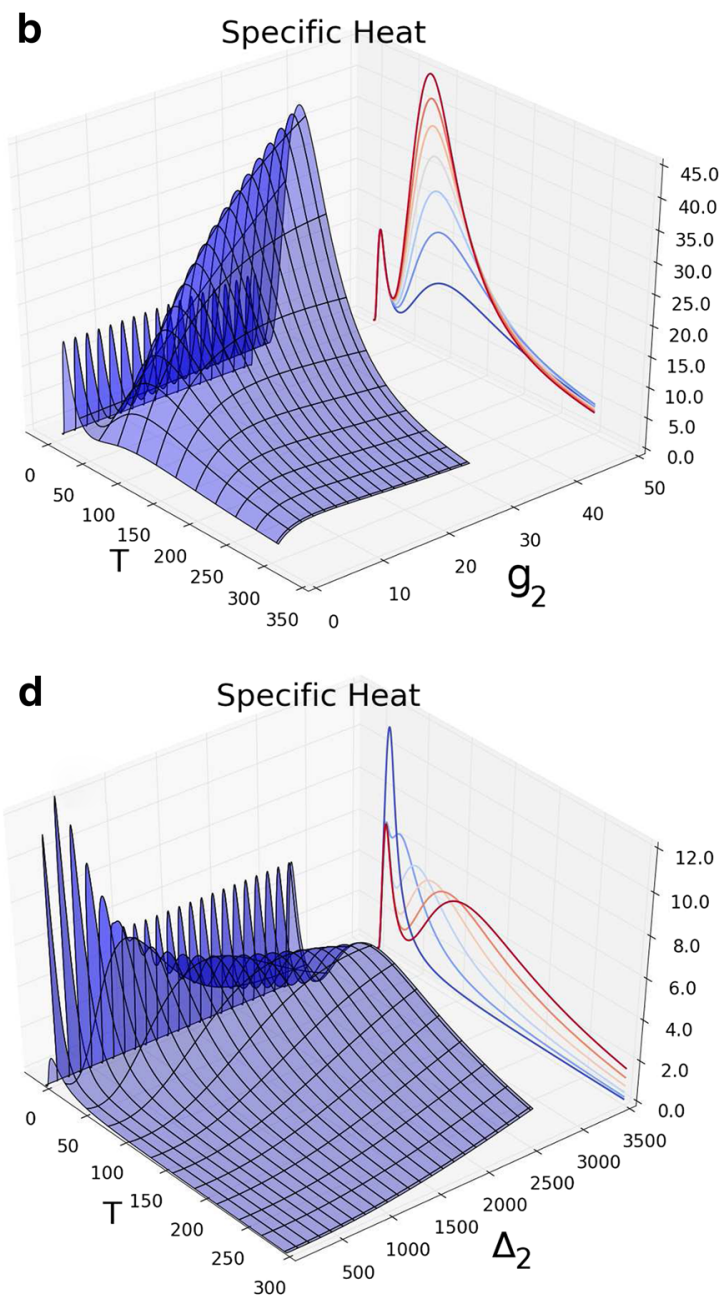

The projection of each curve shown in the $3 \mathrm{D}$ plots is depicted in the various panels. Details are discussed in the main text

characteristic energy scales that differ from each other at least by roughly one order of magnitude. In fact, such features in the specific heat are related to various ongoing topics of interest in condensed matter physics. In particular, $4 f$-electron-based magnetic systems [21] show spectacular properties in this regard.

Hereafter, we focus on examples of various materials classes, in which a two-peak like anomaly in the specific heat versus temperature have been observed experimentally. We start with the heavy-fermion compound $\mathrm{Ce}_{3} \mathrm{Pd}_{20} \mathrm{Si}_{6}$, a magnetic field-induced quantum critical system [23]. For this material, Custers et al. observed the presence of two distinct peaks centered at $T_{Q} \simeq 0.5 \mathrm{~K}$ and $T_{N} \simeq 0.25 \mathrm{~K}$ under an external magnetic field of $0.5 \mathrm{~T}$ in the electronic-specific heat. These authors attributed the features at $T_{Q}$ and $T_{N}$ to a antiferro-quadrupolar magnetic order of the Ce $4 f$ orbitals located on the $8 c$ site and to an antiferromagnetic order, respectively. Yet, the 
authors point out that this scenario is compatible with the $\Gamma_{8}$ quartet and $\Gamma_{7}$ doublet ground states due to the difference of the crystal-field splitting of the Ce atoms on the $4 a$ and $8 c$ lattice sites [24]. The small energy difference of only $\Delta T \simeq 0.25 \mathrm{~K} \simeq 20 \mu \mathrm{eV}$ between the transition temperatures $T_{Q}$ and $T_{N}$ suggests that for $\mathrm{Ce}_{3} \mathrm{Pd}_{20} \mathrm{Si}_{6}$ a subtle change in the system total energy is enough to change the character of the $\mathrm{Ce} 4 f$ orbitals. Interestingly enough, the suppression of $T_{Q}$ gives rise to a magnetic field-induced quantum phase transition. Also, for $\mathrm{CeAuGe}$, $\mathrm{CeCuGe}, \mathrm{CeCuSi}$ [22], $\mathrm{TbPO}_{4}$ [25], $A_{3} \mathrm{Cu}_{3}\left(\mathrm{PO}_{4}\right)_{4}(A=\mathrm{Ca}$, $\mathrm{Sr})$ and $\mathrm{Cu}(3-\mathrm{Chloropyridine})_{2}\left(\mathrm{~N}_{3}\right)_{2}$ [26], $\mathrm{Cu}(\mathrm{en})_{2} \mathrm{Ni}(\mathrm{CN})_{4}$ [27], $\mathrm{URu}_{2} \mathrm{Si}_{2}$ [28] where a magnetic transition followed by the appearance of superconductivity is observed, $\left(\mathrm{Ce}_{1-x} \mathrm{La}_{x}\right)_{3} \mathrm{Al}$ [29], $\mathrm{PrOs}_{4} \mathrm{Sb}_{12}$ [30], and $\mathrm{UPt}_{3}$ [31] with two superconducting transitions, double peaks in the specific have been reported. We now present the results of fits that can be compared to experimental data set for the specific heat of a particular heavy fermion compound. In Fig.4, we show experimental literature results of the electronic-specific heat for CeAuGe [22] together with theoretical fits employing (9). It is worth mentioning that the ordinary phononic contribution to the specific heat was subtracted by the authors of ref.[22], being thus shown in Fig. 4 the bare electronic specific heat contribution originated from the $4 f$-electrons $\left(C_{4 f}\right)$. Interestingly enough, despite the non-interacting gas picture, we have adopted in this work the present approach is capable to describe an electronic system, in which correlation effects are present as discussed above. In order to determine the nature of the electronic/magnetic "excitations" responsible for the emergence of a double-peak anomaly in the specific heat data of $\mathrm{CeAuGe}$, as well for the various materials above-mentioned, microscopic/spectroscopic data are required and constitute a topic out of the scope of the present work. It is worth mentioning that inelastic neutron scattering investigations

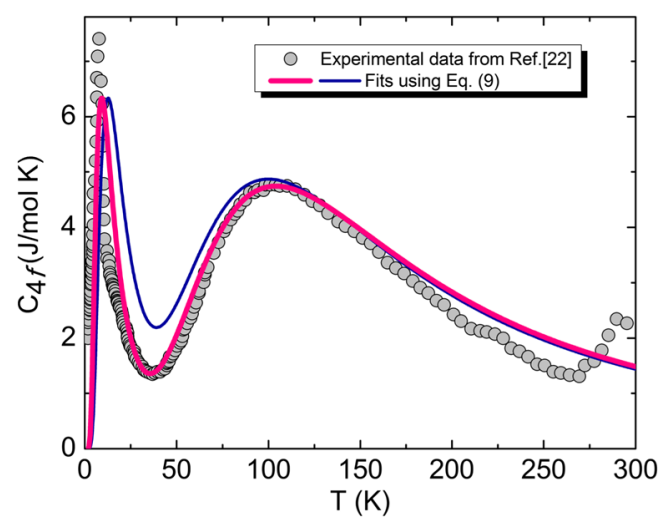

Fig. 4 Electronic-specific heat experimental data $C_{4 f}$ (circles) and theoretical fits (solid lines) for CeAuGe [22] using (9) with $k_{B}=$ $8.314 \mathrm{~J} /(\mathrm{mol} / \mathrm{K}), g_{0}=1, g_{1}=2, g_{2}=4, \Delta_{1}=205 \mathrm{~J} / \mathrm{mol}(282 \mathrm{~J} / \mathrm{mol})$ for the solid pink line (solid blue line) and $\Delta_{2}=2327 \mathrm{~J} / \mathrm{mol}$
[22], carried out on CeAuGe at $15 \mathrm{~K}$, revealed the presence of pronounced crystal field excitations at $24.3 \mathrm{meV}$ ( $\simeq 282 \mathrm{~K}$ ), which roughly corresponds to one of the energy scales, namely $\Delta_{1}=205 \mathrm{~J} / \mathrm{mol}$, employed in one of the fits (solid pink line) depicted in Fig. 4. Note that a fit using $\Delta_{1}=$ $282 \mathrm{~J} / \mathrm{mol}$ (solid blue line in Fig. 4) does not deviate so much from the experimental data. Hence, we figure out that the energy scales $\Delta_{i}$, employed in our model, give us indirect hints of the physical phenomena associated with the specific heat maxima. In general terms, the description of the nature of a phase transition, which usually manifests itself as sharp maximum in the specific heat data (see Fig. 4) in the low-temperature window, as well as the physical mechanism responsible for the broad maximum in the high-temperature range require the combination of complementary experimental techniques. Yet, it is worth mentioning that on the lower temperature flank, namely around the phase transition critical temperature $T_{c}$, the discrepancy between fits and the experimental data can be attributed to the absence of critical fluctuations, see, e.g., Refs. [32-34] and references cited therein, not negligible upon approaching $T_{c}$, in our Ansatz. In the next section, we discuss a degenerate Schottky model.

\section{Degenerate Schottky Model}

Following the proposal of modeling and understanding of the double peak anomalies in specific heat data, in this section, we focus on the description of a degenerate Schottky model. To this end, we consider a two-level system $\left(\varepsilon_{1}=-\frac{1}{2} \Delta, \varepsilon_{2}=\frac{1}{2} \Delta\right.$, with respective degeneracies $\left.\Omega_{1,2}\right)$ plus a spin $\frac{1}{2}$ Zeeman splitting contribution $\pm \mu_{B} H$, cf. scheme shown in the inset of Fig. 5. The partition function and equation for the energy (obtained using (6)) per particle is, disregarding an additive constant, given respectively by [35]:

$$
\begin{aligned}
Z= & \Omega_{1} e^{-\beta \varepsilon_{1}}\left(e^{+\beta m_{0} H}+e^{-\beta m_{0} H}\right) \\
& +\Omega_{2} e^{-\beta \varepsilon_{2}}\left(e^{+\beta m_{0} H}+e^{-\beta m_{0} H}\right), \\
E= & -m H-\frac{\Delta e^{-\beta \Delta}}{\left(1+\Omega e^{-\beta \Delta}\right)},
\end{aligned}
$$

where $\Omega=\Omega_{1} / \Omega_{2}, H$ is an external magnetic field and $\beta$ refers to the inverse temperature. The magnetization $m$ and the specific heat per particle are in turn given respectively by:

$m=\mu_{B} \tanh \left(\beta \mu_{B} H\right)$,

$C_{V}=\frac{\partial E}{\partial T}=-\frac{\partial}{\partial T}(m H)+\frac{\Omega(\beta \Delta)^{2} e^{-\beta \Delta}}{\left(1+\Omega e^{-\beta \Delta}\right)^{2}}$.

For an ordinary paramagnet, cf. discussion in Section 1 (see (3)), the magnetic contribution to the specific heat is 


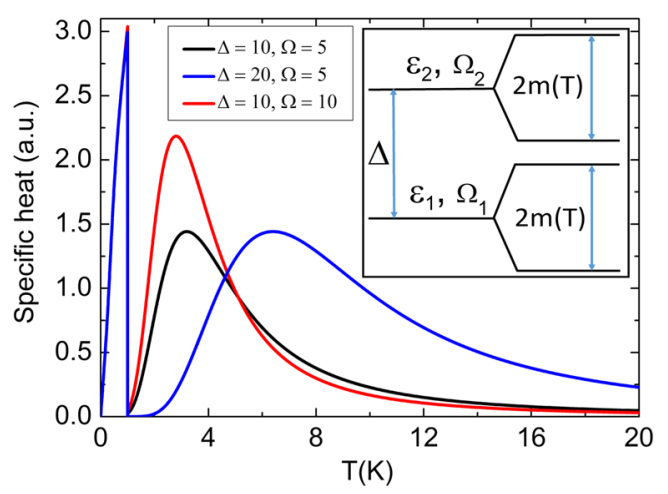

Fig. 5 Main panel: specific heat as a function of temperature for various energy gaps $\Delta$ and degeneracy ratio $\Omega=\Omega_{1} / \Omega_{2}$, cf. indicated in the label. Inset: energy scheme of the degenerate Schottky model. See details in the main text

Schottky type, namely, it is described by the second term of (13) with $\Omega=1$ and an energy gap $\Delta=2 \mu_{B} H$, so that we have two decoupled Schottky like systems. We assume now that a ferromagnetic phase transition, which is a typical many body effect, takes place at a certain critical temperature $T_{\mathrm{c}}$ within the Curie-Weiss molecular mean-field model [35]. We introduce then the macroscopic molecular field phenomenological parameter $\lambda$ and an effective magnetic field $\left(H_{\text {eff }}\right)$, as follows:

$H_{\text {eff }}=H_{\text {ext }}+\lambda m$.

At zero external magnetic field, $m$ is calculated in a selfconsistent fashion via the following equation:

$m=\mu_{B} \tanh \left(\beta \mu_{B} \lambda m\right)$,

being thus now the magnetic gap $\Delta=2 \mu_{B} H=2 \mu_{B} \lambda m$ a function of the order parameter $m$. Scaling the magnetization to $\mu_{B}$, the energies (and temperatures) to $T_{\mathrm{c}}$, with $T_{\mathrm{c}} \equiv \lambda \mu_{B}$, we have:

$C_{V}=-\frac{\partial}{\partial T}\left(m^{2}\right)+\frac{\Omega(\beta \Delta)^{2} e^{-\beta \Delta}}{\left(1+\Omega e^{-\beta \Delta}\right)^{2}}$,

where $m$ is the solution of $m=\tanh (\beta m)$. It is well known that $m \equiv 0$ for $T>1$ and $m(T) \neq 0$ otherwise. Also for small $m$ (for $T$ near and less than 1 ), we have $m^{2} \approx 3(1-T)$ so $C_{V}$ exhibits a jump $\delta C_{V}=3$ at $T=1$. Figure 5 (main panel) depicts the specific heat as a function of temperature for various energy gaps $\Delta$ (in $T_{\mathrm{c}}$ units) and degeneracy ratio $\Omega$, namely, $\Delta=10$ and $\Omega=5$ (black solid line); $\Delta=20$ and $\Omega=5$ (blue solid line); $\Delta=10$ and $\Omega=10$ (red solid line) employing (15). The low- $T$ peak, with a mean-field like shape, remains unaffected upon tuning $\Delta(>>1)$ and $\Omega$. The Schottky maximum, however, becomes broader and shifts to higher temperatures as $\Delta$ is increased, being the peak intensity also increased as $\Omega$ increases cf. expected for a two-level model, a feature that can be understood in terms of entropy arguments as discussed in a previous section. It is worth mentioning that between the ferromagnetic transition and Schottky maximum is exponentially small, i.e., the ferromagnetic phase transition produces a jump in the specific heat data on top of a non-magnetic contribution. For $\Delta$ values close to unity (not shown in Fig. 5), the high- $T$ maximum tail evolves into the low- $T$, being the sharp meanfield like jump $\Delta C=3$ at $T=1$ preserved. Thus, our analysis show that a simple Schottky model coupled to a ferromagnetic phase transition is also amenable to fit emerging double peak/maxima features in measured specific heat data.

\section{Conclusions}

To summarize, we have employed a multilevel model to describe specific heat data as a function of temperature with two maxima. Our results suggest that (i) a ratio of $\Delta_{2} / \Delta_{1} \approx 10$ between the energy levels and (ii) the energy levels degeneracy, govern the two-peaks regime in the specific heat. Apart from the nature of the entities responsible for the emergence of the maxima in the specific heat data, this simple model describes nicely recent literature results for the CeAuGe compound. Furthermore, the specific heat of a degenerate Schottky model was calculated. Our analysis demonstrate that double peak/maxima features in specific heat data can also stem from a two-level model coupled to a ferromagnetic phase transition. We trust that our findings pave the way towards the description of experimental specific heat data for other systems in both soft and condensed matter physics.

Acknowledgments $\mathrm{MdS}$ and RP acknowledge financial support from the São Paulo Research Foundation - Fapesp (Grants No. 365 2011/22050-4 and 2014/15521-9, respectively) and National Council of Technological and Scientific Development - CNPq (Grant Nos. 308977/2011-4 and 305472/2014-3, 308298/2014-4, respectively).

\section{References}

1. M. de Souza, A. Bruehl, J. Mueller, P. Foury-Leylekian, A. Moradpour, J.-P. Pouget, M. Lang, Physica B 404, 494 (2009)

2. R.S. Manna, M. de Souza, A. Bruehl, J.A. Schlueter, M. Lang, Phys. Rev. Lett. 104, 016403 (2010)

3. M. Pregelj, A. Zorko, O. Zaharko, Z. Kutnjak, M. Jagodič, Z. Jagličić, H. Berger, M. de Souza, C. Balz, M. Lang, D. Arčon, Phys. Rev. B 82, 144438 (2010)

4. A. Jesche, C. Krellner, M. de Souza, M. Lang, C. Geibel, Phys. Rev. B 81, 134525 (2010)

5. A. Jesche, T. Förster, J. Spehling, M. Nicklas, M. de Souza, R. Gumeniuk, H. Luetkens, T. Goltz, C. Krellner, M. Lang, J. Sichelschmidt, H.-H. Klauss, C. Geibel, Phys. Rev. B 86, 020501 (2012)

6. R. Lagos, A. Stein-Barana, G. Cabrera, Physica C: Superconductivity 309, 170 (1998)

7. L.A. Macedo, R.E. Lagos, J. Magn. Magn. Mater. 226, 105 (2001) 
8. A. Einstein, Ann. Phys. 22, 180 (1907)

9. P. Foury-Leylekian, S. Petit, I. Mirebeaub, G. Andre, M. de Souza, M. Lang, E. Ressouchee, A. Moradpour, J.-P. Pouget, Phys. Rev. B 88, 024105 (2013)

10. P. Debye, Ann. Phys. 39, 789 (1912)

11. E.S.R. Gopal, Specific Heats at Low Temperatures (Heywood, New York, 1966)

12. R. Pathria, Statistical Mechanics International Series of Monographs in Natural Philosophy (Pergamon Press, 1977)

13. R.P. Hermann, F. Grandjean, G.J. Long, Am. J. Phys. 73, 110 (2005)

14. M. de Souza, J.-P. Pouget, J. Phys.: Condens. Matter 25, 343201 (2013)

15. M. de Souza, A. Brhl, J. Mller, P. Foury-Leylekian, A. Moradpour, J.-P. Pouget, M. Lang, Phys. B Condens. Matter 404, 494 (2009). Proceedings of the International Workshop on Electronic Crystals

16. A. Jesche, C. Krellner, M. de Souza, M. Lang, C. Geibel, New J. Phys. 11, 103050 (2009)

17. L. Landau, E. Lifshitz, Statistical Physics, 5th edn. (Elsevier science, 2013)

18. R.B. Laughlin, D. Pines, Proc. Natl. Acad. Sci. 97, 28 (2000)

19. P.H. Meijer, J.H. Colwell, B. Shah, Am. J. Phys. 41, 332 (1973)

20. A. Abragam, B. Bleaney, Electron Paramagnetic Resonance of Transition Ions International Series of Monographs on Physics (Clarendon, 1970)

21. C. Peiderer, Rev. Mod. Phys. 81, 1551 (2009)

22. B. Sondezi-Mhlungu, D. Adroja, A. Strydom, S. Paschen, E. Goremychkin, Physica B 404, 3032 (2009)
23. J. Custers, K.-A. Lorenzer, M. Mueller, A. Prokofiev, A. Sidorenko, H. Winkler, A. Strydom, Y. Shimura, T. Sakakibara, R. Yu, Q. Si, S. Paschen, Nat. Mater. 11, 189 (2012)

24. T. Goto, T. Watanabe, S. Tsuduku, H. Kobayashi, Y. Nemoto, T. Yanagisawa, M. Akatsu, G. Ano, O. Suzuki, N. Takeda, A. Dnni, H. Kitazawa, J. Phys. Soc. Jpn. 78, 024716 (2009)

25. R. Hill, J. Cosier, S. Smith, Solid State Commun. 26, 17 (1978)

26. T. Nakanishi, S. Yamamoto, Phys. Rev. B 65, 214418 (2002)

27. J. Černák, M. Orendáč, I. Potočňák, J. Chomič, A. Orendáčová, J. Skoršepa, A. Feher, Coord. Chem. Rev. 224, 51 (2002)

28. T. Palstra, A. Menovsky, J.V.D. Berg, A. Dirkmaat, P. Kes, G. Nieuwenhuys, J. Mydosh, Phys. Rev. Lett. 55, 2727 (1985)

29. Y.Y. Chen, Y.D. Yao, B.C. Hu, C.H. Jang, J.M. Lawrence, H. Huang, W.H. Li, Phys. Rev. B 55, 5937 (1997)

30. R. Vollmer, A. Faißt, C. Pfleiderer, H.V. Löhneysen, E.D. Bauer, P.-C. Ho, V. Zapf, M.B. Maple, Phys. Rev. Lett. 90, 057001 (2003)

31. R.A. Fisher, S. Kim, B.F. Woodfield, N.E. Phillips, L. Taillefer, K. Hasselbach, J. Flouquet, A.L. Giorgi, J.L. Smith, Phys. Rev. Lett. 62, 1411 (1989)

32. L. Bartosch, M. de Souza, M. Lang, Phys. Rev. Lett. 104, 245701 (2010)

33. M. de Souza, P. Foury-Leylekian, A. Moradpour, J.-P. Pouget, M. Lang, Phys. Rev. Lett. 101, 216403 (2008)

34. M. de Souza, L. Bartosch, J. Phys.: Condens. Matter 27, 053203 (2015)

35. F. Reif, Fundamentals of Statistical and Thermal Physics (Waveland Press, 2009) 